\title{
Gothic Madwomen: A Comparative Study of Emily Brontë's Wuthering Heights (1847) and Daphne du Maurier's Rebecca
}

\author{
Wang Xinyue
}

UCL, University College London London, UK WC1E 6BT

Email :uclzanq@ucl.ac.uk

\begin{abstract}
ABSRTACT
From the perspective of Feminism and Gothic Studies, this paper Discusses Two novels by famous female writers, Brontë family's Wuthering Heights (1847) and Daphne du Maurier's Rebecca (1938), focus on the two novels in which Catherine and Rebecca two female characters. The traditional Gothic literature presents the female characters in the form of polarization, covering them with the veil of stereotype, which is a great obstacle to discover and convey the true thoughts and feelings of the female characters. However, Catherine used her distinct personality and complex experience to blur the lines drawn by traditional patriarchal culture. Rebecca's tragedy is not due to her Bohemian, but due to the whole Patriarchal Society's heavy restrictions and constraints under the female alienation. The two female characters'deviance is a kind of criticism and Innuendo to the reality, revealing the women's sadness of being bound by marriage and the helplessness of being unable to break away from the control of Patriarchy. They may all be "crazy" women in the secular sense, but their "crazy" is a powerful spiritual strength when they break the shackles placed on women by Patriarchy, which is the best proof of their resistance and struggle.
\end{abstract}

Keywords: Wuthering Heights, Rebecca, female characters, Patriarchy, stereotypes, "crazy" women.

\section{哥特小说中的女性角色刻画}

\author{
王新月
}

英国伦敦大学学院 UCL 英国伦敦 WC1E $6 B T$

Email : uclzanq@ucl.ac.uk

摘要:

本文在女性主义和哥特文学研究的视角下, 探讨两本著名女性作家的小说, 艾米丽 - 勃朗特的《呼啸山庄》 （1847）和达芙妮・杜穆里埃的《蝴蝶梦》(1938), 重点着眼于两本小说当中卡瑟琳和吕蓓卡两位女性角色的塑 造。传统哥特文学以两极分化的形式呈现女性角色, 用刻板印象的面纱罩住她们, 对于发掘和传达女性角色内 心真实想法和感受是一种极大的阻碍。然而卡瑟琳用她鲜明的个性和复杂的体验模糊了传统父权文化给女性 划下的界限。吕蓓卡的悲剧并非源于她的浪荡不羁，而是源于整个父权制社会的重重限制和禁锢下女性的异 化。两位女性角色离经叛道是一种对现实的批判与隐射, 揭露女性被婚姻束缚的悲哀和无法脱离父权制掌控的 无奈。她们也许都是世俗意义上的 “疯” 女人但她们的 “疯” 是在挣脱父权制给女性安上的镣铐时体现出的强 大的精神力量, 是她们的反抗和挣扎的最好证明。

关键词: 《呼啸山庄》; 《蝴蝶梦》; 女性角色塑造; 父权制; 刻板印象; “疯”女人。 作者简介: 王新月, 女, 1998.6.27, 云南麒麟人, 研究生, 主要研究方向比较文学 


\section{1. 新女性的崛起，挣脱父权制镣铐}

传统哥特小说里, 男性角色总能掌控话语权, 男 性角色不论是主角还是反派, 总能把女性角色的命运 窝在手里。女性的形象往往不是人畜无害, 楚楚可怜, 就是放浪形骸, 世俗难容。以两极分化的形式呈现女 性角色，用刻板印象的面纱罩住她们，对于发掘和传 达女性角色内心真实想法和感受是一种极大的阻碍。 Gilbert 的女性主义著作《阁楼上的疯女人: 女性作 家与 19 世纪文学想象》中, 强调文学作品应该发掘 和传达作为女性的真实想法和感受。本文在女性主义 和哥特文学研究的视角下, 探讨两本著名女性作家的 小说, 艾米丽勃朗特的《呼啸山庄》(1847) 和杜穆里 埃的《蝴蝶梦》(1938), 重点着眼于两本小说当中的 女性形象塑造, 用文本细读的方法深入了解女性角色 内心世界, 以及她们精彩的人生体验, 从而致力于把 女性角色从父权主导下的刻板印象中解放出来。

正如学者 Gilbert 注意到的, 《呼啸山庄》中, 女性角色的悲喜好恶对男性角色的幸福安宁有着至 关重要的影响（Gilbert 249）。卡瑟琳显然是全书的 焦点, 她的意志总是那么高昂, 独立又极富个性。《呼 啸山庄》的故事也围绕着卡瑟琳展开。孩童时期的卡 瑟琳在呼啸山庄尽情享受着叛逆无羁的童年, 即便年 纪尚小, 她强烈的自我意识, 和对力量的渴望, 让她 与传统的女性形象划清了界限。一次, 父亲老恩肖问 她想要什么礼物, 卡瑟琳回答, 她想要一根鞭子。不 止是力量与体魄, 通过鞭子, 卡瑟琳想要实现的是对 自己人生的掌控。而男主角老恩肖的养子希克厉的到 来实现了卡瑟琳这一愿望。不仅如此, 希克厉甚至是 卡瑟琳独立与反叛意识的催化剂, 引导她质疑父权制 并拒绝父权制下无权无势的乘巧女儿形象。

希克厉是如何做到的呢? 首先, 老恩肖偏爱希克 厉, 疏离自己的一双儿女, 让卡瑟琳对父亲的所作所 为萌生了质疑。一日, 老恩肖难得地慈爱, 让卡瑟琳 承欢膝下，老恩肖问卡瑟琳：

“卡茜, 你为什么不能永远做一个好姑娘呢”

她就把头抬起来直看着他, 一边笑一边回答

“爸爸, 那你为什么不能永远做一个好男人呢” （勃朗特 52）

卡瑟琳这一铿铭有力的反问显然激起了读者对 于父权制的反思。老恩肖作为父权制当中的掌权人, 他的话语也代表了父权文化对女性的期待。父权制只 想要德艺双馨而且乘顺可人的女性, 但身处权威的人 们从未反思过自身的缺陷与败落。从女性主义的视角 来看, 卡瑟琳对父权制的质问正是她拒绝父权制压迫 与限制, 拒绝父权制为传统女性拷上的枷锁的勇敢尝 试。

希克厉帮助卡瑟琳的改变还体现在他对于老恩 肖和卡瑟琳的截然不同的态度上。老恩肖对希克厉千
依百顺, 宠爱有加, 但希克厉却不冷不热, 爱答不理。 然而, 卡瑟琳却能对他濒指气使, 即便她傲慢无礼, 他也甘之如饴。勃朗特写道: “她的傲慢对于希克厉 可比他的慈爱更有威力……这孩子对于她的话是惟 命是从, 对于他的话却是听得进才听” ( 51) 由此 看来, 卡瑟琳不费吹灰之力, 就能拥有对希克厉至高 的影响力。通常人们认为希克厉是一个不折不扣的混 蛋, 一个毫无同理心的反派角色。但借鉴了Gilbert 的解读, 我认为希克厉是卡瑟琳的鞭子, 是卡瑟琳力 量来源, 由此, 希克厉甚至拥有新男性 (New Man) 的潜质。像新女性 (New Woman) 作家奥莉芙 - 施莱纳 在《妇女与劳动》 (1911) 中提到的那样。新男性是 新女性想要实现性别平等解放女性的最好的陪伴者 和支持者。这样的存在有着动摇传统父权制的强大潜 能, 因为他们理解并支持女性对于独立和力量的向往, 愿意用爱和尊重陪伴女性达成理想。

谈及男性, 本文将对希克厉和埃德加进行对比, 希克厉和埃德加都爱上了卡瑟琳, 但两人的爱有着不 同的原因和目的, 所以比较的意义在于, 通过剖析希 克厉和埃德加对于卡瑟琳不同的态度和期许, 再加上 分析卡瑟琳心中对两人不同的考量, 可以进一步了解 卡瑟琳复杂的内心世界, 探究卡瑟琳追求的究竟是怎 样的自我。埃德加对卡瑟琳的爱始于卡瑟琳被画眉山 庄的斗牛犬咬伤时候。受伤虚弱的卡瑟琳激起了他的 保护欲。那时候埃德加是居高临下的姿态, 卡瑟琳在 他眼里, 就像是奄奄一息的老鼠, 或者是濒死的小鸟 （勃朗特 57）。他爱的是卡瑟琳人畜无害，楚楚可 怜的形象。而希克厉爱的是独立勇敢桀鷔不驯的卡瑟 琳。在《呼啸山庄》中至关重要的一段告白中, 通过 激情澎湃的的宣言 “他就是我自身的存在”（勃朗 特 100）, 卡瑟琳勇敢地表达了内心的真实想法, 那 就是被希克厉爱着的独立勇敢充满野性的她, 才是真 正的卡瑟琳。只有当她的灵魂与希克厉的力量结合, 才是卡瑟琳完整的自我, 一个雌雄同体的、拒绝任何 社会塑造和性别限定的、健全的、独立的个体。由此 梳理埃德加的情感，可以看出埃德加对于希克厉的敌 意不止是源于为同一个女性竞争的敌意,他的敌意更 针对的是卡瑟琳因希克厉而采热的独立意识。由此, 埃德加的敌意也从另一个角度揭示了父权制对于女 性独立意识的不安, 以及对于卡瑟琳所代表的具有颠 覆性的新女性崛起的恐慌。

卡瑟琳最终选择嫁给埃德加是因为和奴仆身份 的希克厉做夫妻, 两人无法维持生计。于是她这样打 算: “要是我嫁给了林墩, 那我就可以帮助希克厉抬 起头来, 安排他从此不再受我哥哥的欺压” (勃朗特 99）。卡瑟琳为了物质上支持希克厉而嫁给埃德加这 一动机一直饱受争议，因为父权制对维多利亚时期的 女性期待往往是 “屋中天使” (Angel in the House) 忠于丈夫, 为家庭无私奉献。然而, 弗吉尼亚 - 伍尔 夫在 “Professions of Women” 一文中提到 “杀死屋 中天使”并塑造有血有肉忠于自我的女性角色是女性 作家最重要的使命（伍尔夫）。如 Mellor 分析的那 
样, 艾米丽・勃朗特呈现给我们的, 是一个模糊了性 别界限的、激情大于理性、野性大于克制的女性角色 （Me11or 192）。卡瑟琳的反叛精神和不屈的意志之 所以会被父权制社会抨击为不知差耻, 正是因为她的 精神具有了对父权制产生动摇的能力。将女性塑造为 天使和荡妇的对立形象是父权制对既存刻板印象的 维护的结果。所以在父权制的话语体系主导下，女性 不是卑微无助, 身若浮萍, 就是心机深沉, 放荡堕落。 也因此前者的命运能够被轻易掌控, 进而安于做 “屋 中天使”，而后者因其强大的精神力量和极具颠覆性 的独立意识, 被父权制视为威胁, 必须抨击、压制、 甚至毁灭。接下来要探讨的《蝴蝶梦》中的吕蓓卡就 是一个典型的例子。因为她强大的鬼力、魄力和影响 力, 招致了杀身之祸。

\section{2. 解析 “疯女人” 女性形象}

吕蓓卡的离经叛道, 比卡瑟琳有过之而无不及。 卡瑟琳曾为了不要成为世俗意义上的随落落魄的女 子, 而压抑自己对希克厉的爱, 陷入无尽的自我折磨 甚至走向精神崩溃。但吕蓓卡只会对世俗虽之以鼻甚 至把世俗规则玩弄于鼓掌之中。吕蓓卡是一个追求性 自由和性解放的新女性, 但也因为她为世俗所不容的 追求, 在父权制的的空洞和邽落蚕食下, 走向了虚伪 和欺骗。吕蓓卡和德温特 - 麦克西姆是世人眼中的模 范夫妇, 一个是十全十美的妻子, 一个是身世显赫的 曼陀丽庄园的主人。殊不知人前神仙滕侣, 人后杀心 四起。迈克西姆对吕蓓卡深恶痛绝, 因为她闪耀华丽 的面具之下, 是永不满足的肉体需求, 和混乱不堪的 私生活。迈克西姆无法揭露吕蓓卡露出真实面目, 因 为吕蓓卡的伪装太过周密, 没有人会相信迈克西姆的 控诉因为 “见过她的人无不以为她是世上心肠最好、 最慷慨大方、最有才华的人” (杜穆里埃 300)。吕 蓓卡的魅力和影响力远远超过了迈克西姆。不甘于做 一个乘顺的 “屋中天使” 的她, 自然成了迈克西姆的 眼中钉, 肉中刺。

迈克西姆控诉道 “吕蓓卡根本不懂得爱, 这女人 没有柔情, 没有起码的是非观, 甚至有点不正常。” (杜穆里埃 300) 这一评价与《呼啸山庄》中的卡瑟 琳太多相似。卡瑟琳告诉女管家纳莉, 要她和希克厉 分开是不可能的, 因为 “即使一切都留下来, 唯独他 毁灭了, 那整个宇宙就变成了一个巨大的陌生人, 我 也不再是它的一部分了”（勃朗特 99）。卡瑟琳与 希克厉的深刻联系也为希克厉出走后之后她的精神 崩溃埋下了伏笔。经历了希克厉锦衣还乡后与埃德加 的剧烈冲突, 埃德加再次要求卡瑟琳在两人中做出选 择, 这时候的卡瑟琳突然毫无道理穷凶极恶地撒野, 甚至自我伤害, 仿佛已经精神错乱。希克厉出于报复 和阴谋与埃德加的妹妹私奔后, 卡瑟琳开始了漫长的 自我囚禁和自我折磨。在心理和生理的双重崩溃之下, 生下小卡瑟琳便去世了。Mellor 认为, 女性在融入父 权主导的家庭生活难以避免要付出生理和心理上的 代价 (Me1lor 10)。无法放弃真实的自我, 同时又强
迫自己做父权制期待的 “好姑娘”。这种矛盾无时无 刻不蚕食着卡瑟琳和吕蓓卡的精神, 从而让她们显得 那么 “不正常”。我认为卡瑟琳和吕蓓卡也许都是世 俗眼中的 “疯” 女人, 但她们并非病理意义上的疯, 她们的 “疯” 是在挣脱父权制给女性安上的镣铐时体 现出的强大的精神力量, 是她们的反抗和挣扎的最好 证明。

不同于卡瑟琳的瞻前顾后, 自我消磨, 吕蓓卡把 她内心难以调和矛盾全部外化为自由放纵。这也是吕 蓓卡这个角色的复杂所在。吕蓓卡甚至野心勃勃地想 要用自己的势力侵占迈克西姆挚爱的曼陀丽庄园, 还 对迈克西姆玩世不恭地说道: “假如我有个孩子....... 不管是你本人还是世上随便哪一个外人, 都将无法证 明孩子不是你生的。小家伙将在曼陀丽长大成人, 姓 你家的贵姓, 到时候你也无计可施啊”（杜穆里埃 309）。吕蓓卡太过聪明，以至于能够熟练地玩弄父 权制长子继承的规则，使其为自己服务。当她生下一 个孩子, 迈克西姆苦心经营维护的一切都将归这个孩 子所有。在这一场对峙中, 吕蓓卡展现了近乎疯狂的 野心。然而最引人深思的是, 虚伪和谎言能够使人在 父权制中获得权力和地位这个事实。正如学者 Auba Llompart Pons 注意到的那样, 不仅是吕蓓卡, 在被 她激怒后杀死吕蓓卡并隐瞒谋杀案的迈克西姆, 还有 帮助迈克西姆隐瞒谋杀真相的迈克西姆的继夫人, 同 样活在谎言和虚伪中。这一切都是为了维护他们在父 权制当中既得的权力和地位（Pons 71）。即便知道 自己的丈夫是一个杀人犯, 德温特夫人不仅没有畏惧 胆寒, 甚至还为自己的丈夫从未爱过吕蓓卡而暗自窃 喜, 毫不犹豫地成为了隐瞒谋杀案的共犯。所以腐坏 良落的不止是吕蓓卡, 更是让她能够肆无忌惮, 并且 让所有人活在虚伪当中的父权制体系。正如哥特研究 学者 Gina Wisker 所言, 吕蓓卡这样的女性并不是红 颜祸水那么简单, 她们的虚伪和值落指引读者剖析更 宏大社会背景，从而揭露父权制更深层的腐坏 (Wisker 85)。

\section{3. 女性 “他者” 的存在形式与颠覆力量}

探讨了女性角色的“疯”所具有的强大精神力量, 分析了吕蓓卡的堕落对父权制体系阴暗面的揭示。接 下来我将探讨女性角色难以捉摸的存在形式以及这 种存在形式所具有的挑战传统父权制设下的边界的 潜能。这一想法的灵感源自 Steven Vine 的文章, 他 认为, 卡瑟琳不论是活着的时候, 还是死去后作为鬼 魂的存在都具有一种“他者”的特质, 并具有一种“呼 啸” (wuther：动摇）的力量（Vine 345）。也就是 说卡瑟琳的存在贯穿全文, 都不断动摇着主导呼啸山 庄的父权制体系。卡瑟琳的鬼魂具有着她孩童时期的 形象, 从弗洛伊德精神分析的角度, 我认为这是一种 精神的回归, 回到了最初凯瑟琳最怀念的叛逆无羁的 孩童时期，也是她所向往的完整的自我。卡瑟琳的鬼 魂不断呼唤着自己的 “鞭子” 也就是希克厉与她的重 逢。希克厉此时通过阴谋和算计成为了呼啸山庄和画 
眉山庄的主人。他拥有着并折磨着曾经把他踩在脚下 的一切。唯独不能再次拥有卡瑟琳。处于父权制权威 的希克厉, 似乎完全忘记了自己也曾是父权制下的受 害者。然而卡瑟琳的鬼魂, 正是动摇他被复仇蒙蔽的 内心的关键所在。正是卡瑟琳的鬼魂不断翟痛着他的 良心, 刺激着他的神经, 让他明白原来自己所谋求来 的一切都是一片虚无。哥特文学中的鬼魂, 往往具有 跨越边界的潜能, 介于存在与不存在之间的鬼魂, 盘 桓在《呼啸山庄》和《蝴蝶梦》的世界, 动摇着既存 的秩序, 纠缠着每一个角色的内心。

在《蝴蝶梦》中, 以鬼魂的形式存在的吕䔒卡同 样拥有着她无所不在的微妙而又深刻的影响力。让无 名的叙述者德温特夫人从一个乘顺的孩子气的女性 转变为一个具有颠覆性的独立的个体。德温特夫人是 一个刚毕业的身世低微的年轻女性, 她与迈克西姆的 相爱, 虽然表面是灰姑娘故事的模板, 但实则错综复 杂扑朔迷离。在知道真相之前, 她自卑怯懦, 认为自 己无论如何也无法超越死去的吕蓓卡在丈夫心中的 地位。每一个人都爱着怀念着吕蓓卡, 虽然她才是曼 的利庄园的女主人, 但她卑微透明的存在让她比吕蓓 卡更像一个鬼魂。她的精神被吕蓓卡的鬼魂折磨着, 同时也被潜移默化地改变着（Sivinski 861）。本来 对丈夫言听计从的她, 在迈克西姆去伦敦时, 感慨道 “一种无拘无束的自由感在心头油然而生! 大有无牵 无挂一身轻的味道”（杜穆里埃 163）之后她警觉 自己竟然会有如此 “大不敬” (163) 的念头。由此 可见吕蓓卡微妙的影响力已经渗透了她的内心, 让她 从一个不经世事的女性, 转变为一个具有独立意志和 思想的个体。

\section{4. 结论}

本文重点探索了两位饱受争议的女性角色的内 心世界, 以及她们的行为动机。值得一提的是《蝴蝶 梦》情节的扑朔迷离, 其实就在于读者其实并不能真 正地探知真相, 关于吕蓓卡的放荡和陏落, 全都来自 迈克西姆的一家之言。结局的大火烧光了富丽堂皇的 曼陀丽庄园, 也烧光了吕蓓卡存在的一切印记, 但是 作为谋杀共犯的德温特夫妇真的能够如童话般幸福 地生活下去吗？被吕蓓卡唤醒了内心独立意志的德 温特夫人, 真的不会成为下一个吕蓓卡吗? 这些都不 得而知, 留给我们的思考就是, 卡瑟琳或是吕蓓卡并 不是理想中的完美女性, 只能赞美不能批判。但是, 在探讨哥特文学尤其是其中女性角色的刻画时, 应该 着眼于她们丰满而复杂的心理旅程和人生体验, 从而 支持女性敢于表达自我, 遵循自己的内心的真理, 而 不因父权制的期待和束缚压迫自我。

\section{REFERENCES}

[1] Gilbert Sandra M. et al. "Looking Oppositely: Emily Brontë's Bible of Hell." The Madwoman in the Attic: The Woman Writer and the Nineteenth-Century
Literary Imagination, Yale University Press, NEW HAVEN; LONDON, 2020, pp. 248-308. .

Mellor, Anne K. Romanticism and Gender, Routledge, 1993.

[2] Schreiner, Olive. Woman and Labour. A free ebook from http://manybooks.net/

Pons, Auba Llompart. "Patriarchal Hauntings: Re-Reading Villainy and Gender in Daphne Du Maurier's 'Rebecca'.' Atlantis, vol. 35, no. 1, 2013, pp. 69-83.

[3] Sivinski, Stacy. "An invention...that bottled up a memory, like scent": phantom fragrances in Daphne du Maurier's Rebecca, Journal of Gender Studies, 29:8, 2020, pp. 860-869.

[4] Vine, Steven. "The Wuther of the Other in Wuthering Heights." Nineteenth-Century Literature, vol. 49, no. 3 , 1994, pp. 339-359.

[5] Wisker, Gina. "Dangerous Borders: Daphne du Maurier's Rebecca: Shaking the foundations of the romance of privilege, partying and place.” Journal of Gender Studies, 12:2, 2003, pp. 83-97.

[6] Woof, Virginia. "Professions of Women." Selected Essays (Oxford World's Classics) Edited with an Introduction and Notes by David Bradshaw, Oxford University Press, 2008.

[7] Brontë family, emily, Wuthering Heights by Shanghai Translation Publishing House Henry Fong

[8] Du Maurier, Daphne, butterfly dreams, Lin Zhiling, Shanghai Translation Publishing House 